\title{
Surface engineered antifouling optomagnetic SPIONs for bimodal targeted imaging of pancreatic cancer cells
}

This article was published in the following Dove Press journal:

International Journal of Nanomedicine

27 March 2014

Number of times this article has been viewed

\author{
Xiaohui Wang' \\ Xiaohong Xing' \\ Bingbo Zhang' \\ Fengjun Liu' \\ Yingsheng Cheng ${ }^{2}$ \\ Donglu Shi ${ }^{1,3}$
}

'Radiology Department of the Tenth People's Hospital,The Institute for Biomedical Engineering and Nano Science, Tongji University School of Medicine, Shanghai, People's Republic of China; ${ }^{2}$ Department of Radiology, Shanghai Sixth People's Hospital, Shanghai Jiaotong University, Shanghai, People's Republic of China; ${ }^{3}$ Materials Science and Engineering Program, Department of Mechanical and Materials Engineering, College of Engineering and Applied Science, University of Cincinnati, Cincinnati, $\mathrm{OH}$, USA
Correspondence: Bingbo Zhang Tongji University, Mailbox No I55, 1239 Siping Road, Shanghai, 200092, People's Republic of China Tel +86 2I 65983706819 Fax +8621 659837060 Email bingbozhang@tongji.edu.cn

Yingsheng Cheng

Shanghai Sixth People's Hospital,

Room 50I,Teaching Building,

600 Yishan Road, Shanghai,

People's Republic of China

Tel +86 2I 24058068

Fax +86 21 38297858

Email cjr.chengysh@vip.163.com

\begin{abstract}
Targeted imaging contrast agents for early pancreatic ductal adenocarcinoma diagnosis was developed using superparamagnetic iron oxide nanoparticles (SPIONs). For phase transfer of SPIONs, the hydrophobic SPIONs are first treated with tetrafluoroborate and then capped by bovine serum albumin (BSA) via ligand exchange. It was experimentally found that nitrosyl tetrafluoroborate pretreatment and proper structures of molecules are essential to the effective surface functionalization of SPIONs. Nonspecific binding was found to be significantly reduced by BSA surface functionalized hydrophobic SPIONs (BSA-SPIONs). The BSA.SPIONs were monodispersed with an average size of approximately $18.0 \mathrm{~nm}$ and stable in a wide $\mathrm{pH}$ range and various ionic strengths even after 7 days of storage. The longitudinal and transverse proton relaxation rate $\left(\mathrm{r}_{1}, \mathrm{r}_{2}\right)$ values of the BSA.SPIONs were determined to be 11.6 and $154.2 \mathrm{~s}^{-1}$ per $\mathrm{mM}$ of $\mathrm{Fe}^{3+}$ respectively. The $\mathrm{r}_{2} / \mathrm{r}_{1}$ ratio of 13.3 ensured its application as the $\mathrm{T}_{2}$-weighted magnetic resonance imaging contrast agents. When conjugated with near-infrared fluorescent dye and monoclonal antibody, the ${ }^{\text {dye }}$ BSA.SPION-monoclonal antibody bioconjugates showed excellent targeting capability with minimal nonspecific binding in the bimodal imaging of pancreatic cancer cells. The experimental approach is facile, environmentally benign, and straightforward, which presents great promise in early cancer diagnosis.
\end{abstract}

Keywords: superparamagnetic iron oxide nanoparticles, BSA, bimodal imaging, MRI, targeted imaging

\section{Introduction}

Pancreatic ductal adenocarcinoma (PDAC), as a major disease with an extremely dismal 5 -year survival rate below 5\%, has attracted great attention not only in the medical communities but also in general public around the world. ${ }^{1,2}$ Due to lack of accurate diagnostic tools, the majority of patients with advanced disease or metastasis exhibit considerable complications in surgery. Therefore, developing novel methods for early diagnosis is essential to improvement the PDAC survival rate.

Nano contrast agent (CA) based imaging with targeted functionalities show promise in early cancer diagnosis. Among the most commonly used nano CAs, superparamagnetic iron oxide nanoparticles (SPIONs) are of particular interest for their good biocompatibility, superior magnetic resonance (MR) $\mathrm{T}_{2}$ (transverse relaxation) shortening effects, and biodegradability. ${ }^{3-6}$ Furthermore, SPIONs exhibit large surface areas, making them favorable for versatile surface functionalization and conjugation of biomolecules. ${ }^{7,8}$ Two most extensively used methods in preparing SPIONs for magnetic resonance imaging (MRI) are coprecipitation and thermal decomposition. The coprecipitation technique is probably the simplest chemical route in the preparation 
of hydrophilic SPIONs. But this method does not provide good control on size distribution and particle geometry. In contrast, thermal decomposition in the oil phase can produce high quality hydrophobic iron oxide nanoparticles with perfect monodispersity and high crystallinity by modulating reaction temperature, reaction time, surfactant/precursor concentration, and type of solvent. ${ }^{9}$ However, the obtained nanoparticles are usually hydrophobic and dispersible in organic solvent which requires further phase transfer procedures to make them water soluble. Various methods can be used for this phase transfer, such as polymer coating, ${ }^{10}$ surfactant adsorption/exchange, ${ }^{11}$ and silanization. ${ }^{12}$ However, nonspecific binding is still a critical challenge for targeted diagnosis. ${ }^{13,14}$ Nonspecific interaction of SPIONs with normal cell membranes may cause inefficient tagging to the desired targets, resulting in a high level of background signal that severely limits the contrast and sensitivity of the diagnostic imaging. Previous studies have shown that neutral and negatively charged SPIONs exhibit significant antifouling that can effectively reduce nonspecific binding. ${ }^{15,16}$ Polyethylene glycol (PEG), known for its hydrophilic nature and biocompatibility, is often used to modify the surface properties of the nanoparticles for reduction of nonspecific binding and enhancement of colloidal stability and biocompatibility. ${ }^{17,18}$ However, PEG surface functionalization decreases the volume fraction of magnetite, leading to reduced overall magnetic response of SPIONs. ${ }^{19}$ The surface modification processes are also generally tedious, high cost, and environmentally unfriendly. It is, therefore, important to seek for other surface engineering routes with combined advantages in targeted imaging for early cancer diagnosis.

Bovine serum albumin (BSA) has been extensively used in biological applications due to its capability of reducing nonspecific binding in immunoassay, ${ }^{20}$ commercial viability, and low cost. BSA is a zwitterionic surfactant with abundant carboxyl and amino groups. Such a unique structure not only provides sufficient binding sites for further functionalization, but also imparts good colloidal stability in both acidic and basic environments. In our previous work, we used BSA as the stabilizer and capping biopolymer, and successfully transferred hydrophobic quantum dots (QDs) and SPIONs into the hydrophilic phase under ultrasonication conditions. ${ }^{21,22}$ The BSA coated QDs exhibited excellent antifouling property. The only difference found between the SPIONs and QDs was that the former clustered considerably while the latter individually dispersed in water solution. This is mainly attributed to the varied affinity of BSA from QDs to SPIONs. ${ }^{21,22}$ The SPIONs formed clusters of nearly $100 \mathrm{~nm}$ and showed appreciable MRI as a result of high uptake in liver. However, for the nonreticuloendothelial system, the nanoparticles must be kept small enough to escape the reticuloendothelial system for prolonged blood circulation time. ${ }^{23}$

Protein-based surface modification has been studied by a number of groups and shows advantages in biocompatibility, colloidal stability, high payload, and postmodifications. ${ }^{24-26}$ As shown in Figure 1, a novel synthesis route is developed based on BSA surface engineering, aiming at procuring small (below $20 \mathrm{~nm}$ ) and antifouling monodisperse magnetic nanoparticles with minimal nonspecific binding. Before BSA surface functionalization, the hydrophobic SPIONs are treated with tetrafluoroborate $\left(\mathrm{NOBF}_{4}\right) . \mathrm{NOBF}_{4}$ treatment is a transition phase, having great advantages when compared to direct BSA surface functionalization of SPIONs in our previous work. ${ }^{22}$ The purpose of this new approach is to exchange the organic ligands on SPIONs by $\mathrm{NOBF}_{4}$. The $\mathrm{NOBF}_{4}$-stabilized SPIONs are subsequently functionalized by BSA. This method is experimentally facile, effective, and reproducible. The as-prepared BSA surface functionalized hydrophobic SPIONs (BSA·SPIONs) exhibit good colloidal stability and high $\mathrm{r}_{2}$ relaxivity. Upon conjugation with $\mathrm{Cy} 5$ dye (for near-infrared fluorescence [NIRF] imaging) and anti-plectin-1 monoclonal antibody $(\mathrm{mAb})$, the resulting

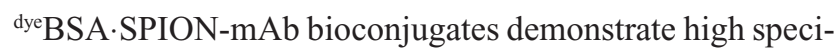
ficity in recognition of plectin-1, a specific biomarker for PDAC, ${ }^{27,28}$ in fluorescent and MR bimodal imaging.

\section{Materials and methods Materials}

All reagents were purchased from Sigma-Aldrich (St Louis, MO, USA) and available commercially. Ultrapure Millipore deionized water $\left(18.2 \mathrm{M} \Omega \cdot \mathrm{cm}\right.$ resistivity at $\left.25^{\circ} \mathrm{C}\right)$ was used throughout the experiments. Human PDAC cell lines Panc-1 and normal L02 cells were purchased from Cell Bank of the Chinese Academy of Sciences (Beijing, People's Republic of China) and cultured according to the established protocols.

\section{BSA surface engineering of SPIONs}

Hydrophobic SPIONs were synthesized according to a previously reported procedure. ${ }^{9}$ BSA-stabilized SPIONs (BSA.SPIONs) were prepared by a two step process. The first step was $\mathrm{NOBF}_{4}$ treatment on hydrophobic SPIONs aimed at exchanging the organic ligands of SPIONs. This procedure was performed according to a previously described method with slight modifications. ${ }^{29}$ Briefly, $2 \mathrm{~mL}$ of SPIONs dispersion in n-heptane (approximately $5 \mathrm{mg} / \mathrm{mL}$ ) was mixed with $10 \mathrm{~mL}$ of trichloromethane solution of $\mathrm{NOBF}_{4}(0.01 \mathrm{M})$ 


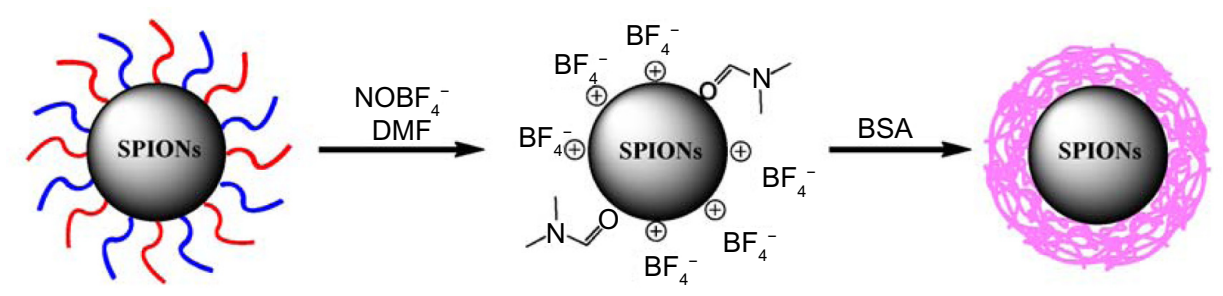

Hydrophobic SPIONs

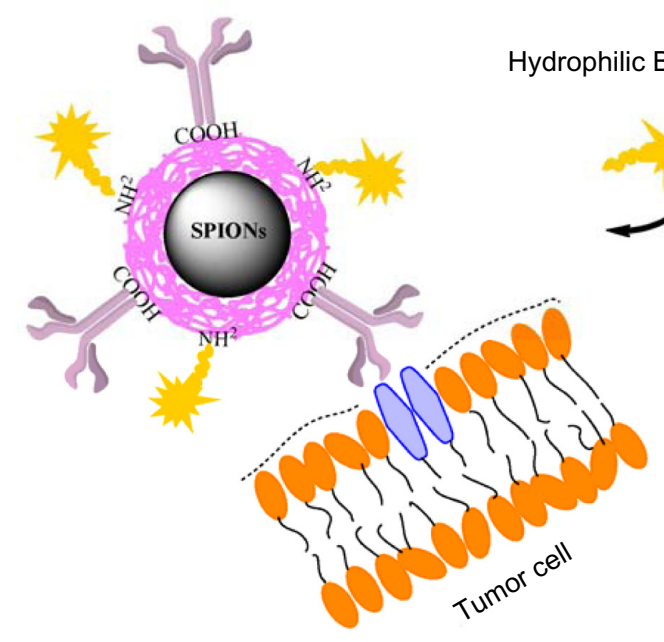

Figure I Schematic illustration of dyebovine serum albumin surface functionalized hydrophobic superparamagnetic iron oxide nanoparticles-monoclonal antibody bioconjugates.

Abbreviations: BSA, bovine serum albumin; BSA.SPIONs, bovine serum albumin surface functionalized hydrophobic superparamagnetic iron oxide nanoparticles; DMF, dimethylformamide; mAb, monoclonal antibody; $\mathrm{NOBF}_{4}$, tetrafluoroborate; SPIONs, superparamagnetic iron oxide nanoparticles.

at room temperature. The resulting mixture was shaken gently until the precipitation of SPIONs was observed, typically within 5 minutes. After centrifugation to remove the supernatant, the precipitated SPIONs were redispersed in $2 \mathrm{~mL}$ of dimethylformamide (DMF) to form a stable colloidal dispersion. The second step was BSA surface functionalization. BSA $(10 \mathrm{mg}$ ) was dissolved completely in $2 \mathrm{~mL}$ of deionized water and added into the above SPIONs/DMF solution followed by vigorous stirring for 2 hours at room temperature. The solution was then added into a dialysis bag (molecular weight cutoff of 8,000 Da to approximately $14,000 \mathrm{Da}$ ) and the dialysis bag was immersed in deionized water under shaking to displace DMF. The resulting aqueous solution was purified via ultracentrifugation twice $(100,000 \times g, 30$ minutes $)$ to remove residual BSA. The final purified BSA.SPIONs were dispersed in borate saline buffer ( $50 \mathrm{mM}, \mathrm{pH} 8.2$ ).

\section{Preparation of dyeBSA.SPION conjugates and dyeBSA.SPION-mAb bioconjugates}

The nontargeted ${ }^{\text {dye }} \mathrm{BSA}$.SPION NIRF/MR bimodal imaging CAs were prepared by Cy5-mono N-hydroxysuccinimide ester directly reacting with the amino group on the BSA.SPIONs overnight in borate saline buffer $(50 \mathrm{mM}, \mathrm{pH} 8.2)$ at room temperature. The excess $\mathrm{Cy} 5$ dye was removed by dialysis with a dialysis bag (molecular weight cutoff 8,000 Da to approximately $14,000 \mathrm{Da})$ against borate saline buffer $(50 \mathrm{mM}$, $\mathrm{pH}$ 8.2). The conjugation of Cy5 dye to the BSA.SPIONs was determined by fluorescence spectroscopy.

The targeted ${ }^{d y}$ BSA.SPION-mAb bioconjugates were prepared by a carbodiimide reaction linking anti-Plec-1 antibodies to the purified dyeBSA.SPION conjugates using ethyl-3-(3-dimethylaminopropyl)carbodiimide hydrochloride $(\mathrm{EDC} \cdot \mathrm{HCl})$ as the crosslinker. The ${ }^{d y e} \mathrm{BSA} \cdot \mathrm{SPIONs}$ were reacted with monoclonal antibody at an appropriate $\mathrm{Fe}^{3+} / \mathrm{mAb} / \mathrm{EDC} \cdot \mathrm{HCl}$ molar ratio in borate saline buffer ( $50 \mathrm{mM}, \mathrm{pH} 8.2$ ) for 2 hours at room temperature. The final bioconjugates were dispersed in phosphate buffered saline ([PBS] $0.01 \mathrm{M}, \mathrm{pH} 7.4,0.5 \% \mathrm{BSA}, 0.02 \%$ sodium azide) after being purified by ultracentrifugation at $100,000 \times g$ for 15 minutes and washed with 0.01 M PBS ( $\mathrm{pH} 7.4$ ) twice. The conjugation of anti-Plec-1 antibodies was confirmed using a test trip on the basis of the primary and secondary antibody response. ${ }^{30}$

\section{Material characterization and statistical analysis}

Transmission electron microscopy (TEM) was carried out on a JEOL JEM-1230 TEM (JEOL, Tokyo, Japan) with a tungsten 
filament at an accelerating voltage of $200 \mathrm{kV}$. The sample was prepared by placing a drop of prepared sample on the surface of copper grids and dried at room temperature. Fourier transform infrared spectra (FTIR) were obtained on a TENSOR 27 FTIR spectrometer (Bruker Corporation, Billerica, MA, USA) over a potassium bromide pellet. The samples dispersed in different solutions (chloroform for SPIONs, DMF for NOBF4-SPIONs, water for BSA and BSA.SPIONs) were dried before FTIR analysis. The crystalline structures of the as-prepared samples were evaluated by X-ray diffraction (XRD) analysis on a DX-1000 diffractometer (Dandong Fangyuan Instrument Co., Ltd., Dandong, People's Republic of China) by using $\mathrm{CuK}_{\alpha}$ radiation $(\lambda=0.15406 \mathrm{~nm})$. The operation tube voltage and current were kept at $40 \mathrm{kV}$ and $30 \mathrm{~mA}$, respectively. Dynamic light scattering (DLS) data of the samples in borate buffer was taken using a particle size analyzer (Nano ZS90; Malvern Instruments, Malvern, UK). Laser scanning confocal images were collected on a laser scanning confocal microscope (Leica TCS SPS; Leica Microsystems, Wetzler, Germany). Emission spectra were observed on a LS-55 spectrophotometer (Perkin Elmer, Waltham, MA, USA). The magnetic characterization was carried out with a vibrating sample magnetometer on a LDJ9600-1 physical property measurement system (LDJ Electronics, Troy, MI, USA) at $300 \mathrm{~K}$. Statistical analysis was performed using SPSS18.0 (SSPS Inc., Chicago, IL, USA).

\section{Colloidal stability study of BSA.SPIONs}

The prepared BSA.SPIONs were dissolved in various $\mathrm{pH}$ buffer solutions ( $\mathrm{pH}$ value varied from 4 to 13 ) and sodium chloride water solutions (ionic strength varied from $0.01 \mathrm{M}$ to $1 \mathrm{M}$ ) for 7 days at room temperature. The DLS analyses were performed to evaluate the hydrodynamic diameters and zeta potentials of the samples in every solution.

\section{In vitro relaxometry}

The longitudinal and transverse relaxation times were determined using a $1.41 \mathrm{~T}$ minispec mq $60 \mathrm{NMR}$ Analyzer (Bruker Optik $\mathrm{GmbH}$, Ettlingen, Germany) at $37^{\circ} \mathrm{C}$. The relaxivity values of $\mathrm{r}_{1}$ and $r_{2}$ were calculated by fitting the $1 / \mathrm{T}_{1}$ and $1 / \mathrm{T}_{2}$ relaxation time $\left(\mathrm{s}^{-1}\right)$ versus $\mathrm{Fe}^{3+}$ concentration $(\mathrm{mM})$ curves. The in vitro $\mathrm{MR}$ images of BSA.SPIONs were obtained using a head coil of Siemens MRI system (MAGNETOM Verio; Siemens Healthcare, Munich, Germany). The measurement conditions were as follows: $\mathrm{T}_{2}$-weighted sequence, multislice spin echo, repitition time/ echo time $(\mathrm{TR} / \mathrm{TE})=4,800 / 80 \mathrm{~ms}$, matrix acquisition $=134 \times 192$, number of excitations $(\mathrm{NEX})=3$, field of view $(\mathrm{FOV})=63 \mathrm{~mm} \times$ $63 \mathrm{~mm}$, FOV phase of $100 \%$, thickness $=2.5 \mathrm{~mm}, 3 \mathrm{~T}, 25^{\circ} \mathrm{C}$.

\section{In vitro laser scanning confocal imaging and MRI}

Panc-1 cell lines (Plec-1 overexpressed) ${ }^{27}$ were used in this study to evaluate the specificity of the dye BSA.SPION-mAb bioconjugates. Cells were cultured in Dulbecco's Modified Eagle's Medium (high glucose) mixture with 10\% fetal bovine serum and penicillin/streptomycin $(100 \mathrm{U} / \mathrm{mL})$ at $37^{\circ} \mathrm{C}$ in a humidified atmosphere containing $5 \% \mathrm{CO}_{2}$. Cells were plated in glass-bottomed microwell dishes and cultured for 24 hours. Following this, $2 \mathrm{mM}\left(\mathrm{Fe}^{3+}\right)$ of Plec-1-

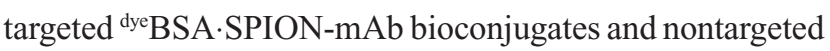
${ }^{d y e}$ BSA.SPION conjugates was added into the Panc-1 cells, respectively, and incubated with the corresponding cells for 5 minutes. After being washed twice with $0.01 \mathrm{M}$ PBS, the cells were imaged under a laser scanning confocal microscope (Leica TCS SPS; Leica Microsystems).

For in vitro cell MRI experiments, the cells were

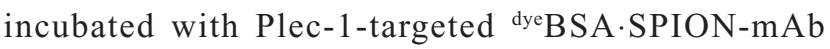
bioconjugates and nontargeted ${ }^{\text {dye }} \mathrm{BSA} \cdot \mathrm{SPION}$ conjugates, respectively, at $37^{\circ} \mathrm{C}$ for 2 hours. Cells were washed and then redispersed in eppendorf tubes. The tubes were scanned in a 3 T MRI scanner (MAGNETOM Verio; Siemens Healthcare) using multiecho $\mathrm{T}_{2}$ weighted fast-spin echo imaging sequences (head coil, TR/TE $=4,800 / 80 \mathrm{~ms}$, matrix acquisition $=128 \times 128, \mathrm{NEX}=3, \mathrm{FOV}=60 \times 60 \mathrm{~mm}$, FOV phase of $100 \%$, thickness $=2 \mathrm{~mm}, 3 \mathrm{~T}, 25^{\circ} \mathrm{C}$ ).

To evaluate the targeting capability of ${ }^{\text {dye }} \mathrm{BSA} \cdot \mathrm{SPION}-$ $\mathrm{mAb}$ bioconjugates, a competitive inhibition cell assay was performed in both fluorescent and MR imaging by adding free Plec-1 monoclonal antibodies $(10 \mu \mathrm{L}, 0.1 \mathrm{mg} / \mathrm{mL})$ into Panc-1 cells for preincubation before introduction of the Plec-1-targeted dye BSA.SPION-mAb bioconjugates.

\section{Cytotoxicity assay}

In vitro cytotoxicity was assessed on L02 cells, using a modified 3-(4, 5-dimethylthiazol-2-yl)-2, 5-diphenyltetrazolium bromide (MTT) method. ${ }^{31}$ Before application of the surface saturated SPIONs to the MTT assays, some necessary steps were performed including introduction of the nanoparticles into the cell medium and leaving the solution in contact for a period of 24 hours, and then replacing with a fresh medium. Cells $(100 \mu \mathrm{L})$ growing in the log phase were seeded in a 96-well flat culture plate at $1 \times 10^{4}$ cells per well and subsequently incubated for 24 hours at $37^{\circ} \mathrm{C}$ under $5 \% \mathrm{CO}_{2}$. The pretreated BSA.SPIONs samples with different concentrations were added to each group (six wells) for 24 hours. MTT solution $(10 \mu \mathrm{L}, 5 \mathrm{mg} / \mathrm{mL}$ in PBS $)$ was added to the wells and 
incubated for an additional 4 hours. MTT internalization was terminated by aspiration of the media, and the cells were lysed with dimethylsulfoxide $(150 \mu \mathrm{L} /$ well $)$. The absorbance of the suspension was measured at $570 \mathrm{~nm}$ (intensity of absorbance [IA] value) on a Tecan Infinite M200 monochromator-based multifunction microplate reader (Tecan Group Ltd, Mannedorf, Switzerland) with background subtraction at $690 \mathrm{~nm}$. The following formula was used to calculate the viability of cell growth: cell viability $(\%)=($ mean of IA value of treatment group/mean of IA value of control $) \times 100$.

\section{Results and discussion Phase transfer of SPIONs}

BSA is a water soluble macromolecule containing one single cysteine and eight pairs of disulfide bonds. ${ }^{32}$ These sulfhydryl compounds can act as multidentate ligands that replace the original ones on some hydrophobic nanoparticles via ligand exchange. ${ }^{33-35}$ Based on the unique structure of BSA, it was found that the QDs were effectively surface functionalized under ultrasonication. ${ }^{21}$ However, SPIONs were found to cluster at an average size of $100 \mathrm{~nm} .^{22}$ In this study, a transition process by $\mathrm{NOBF}_{4}$ was introduced to the surface functionalization of SPIONs.

Figure 1 illustrates the synthesis route of dye BSA.SPION$\mathrm{mAb}$ bioconjugates. BSA.SPIONs are designed as the nano carrying systems for construction of the ${ }^{d y}$ BSA.SPION and ${ }^{d y}$ BSA.SPION-mAb conjugates. The originally synthesized SPIONs are hydrophobic and only soluble in nonpolar solvents such as n-heptane due to their surface organic ligands. As shown in Figure 1, the first treatment involves adding $\mathrm{NOBF}_{4} / \mathrm{DMF}$ to the SPION/n-heptane solution. After gentle shaking of the mixture solution, flocculation is observed, indicating solubility change of SPIONs as a result of $\mathrm{NOBF}_{4}$ surface modification. After slight centrifugation to remove the supernatant, the precipitated SPIONs can be readily redispersed in various hydrophilic solvents such as DMF without precipitation.

The hydrophilic nature suggests the removal of the original organic ligands, which was further verified by FTIR analysis (Figure S1). The FTIR spectra of SPIONs show the intensity of the characteristic $\mathrm{C}-\mathrm{H}$ stretching vibrations around 2,800 $\mathrm{cm}^{-1}$ ascribed to oleic acid molecules. A new peak at $1,028 \mathrm{~cm}^{-1}$ in the $\mathrm{NOBF}_{4} \cdot \mathrm{SPIONs}$ spectrum, which is assigned to $\mathrm{BF}_{4}{ }^{-}$anions, ${ }^{36}$ suggests an exchange between the organic ligands and inorganic $\mathrm{BF}_{4}^{-}$anions. Another peak around $1,630 \mathrm{~cm}^{-1}$ can be attributed to $\mathrm{C}=\mathrm{O}$ stretching vibrations of the solvent DMF molecules. ${ }^{29}$ The remaining broad band around $3,500 \mathrm{~cm}^{-1}$ is attributable to the solvated water molecules, accordant with the hydrophilic nature of the SPIONs dispersion.

It is worth noting that dispersing $\mathrm{NOBF}_{4} \cdot \mathrm{SPIONs}_{\mathrm{N}}$ in water results in partial aggregations, even though $\mathrm{NOBF}_{4} \cdot \mathrm{SPIONs}$ have high solubility in DMF, a polar (hydrophilic) aprotic solvent miscible with water and majority of organic liquids. It indicates the poor compatibility of $\mathrm{NOBF}_{4} \cdot \mathrm{SPIONs}_{\mathrm{N}}$ with water molecules, an issue that has to be addressed for biological applications. Hydrophilic BSA protein was selected for further surface treatment of $\mathrm{NOBF}_{4} \cdot \mathrm{SPIONs}$. This step was important for stabilizing SPIONs in water without precipitation and reducing nonspecific cellular binding.

Upon BSA surface functionalization, SPIONs were found to disperse well in water without aggregation. The dispersion is associated with the successful coating of BSA via secondary ligand exchanging (Figure 1). FTIR analysis provides the evidence of BSA on the SPIONs surfaces (Figure S1). The BSA.SPIONs exhibit two additional bands at $1,350 \mathrm{~cm}^{-1}$ and $1,650 \mathrm{~cm}^{-1}$ which are identical to those on pure BSA. The reduced peak at $1,028 \mathrm{~cm}^{-1}$ indicates the removal of $\mathrm{BF}_{4}^{-}$anions on the surface of SPIONs. In BSA surface functionalization, removal of DMF is found to be necessary for the formation of stable BSA.SPIONs in water solution. As shown in Figure S2, the BSA.SPIONs dialyzed against deionized water can be well dispersed in deionized water and the dispersion is optically clear without obvious aggregates or sediments after 7 days of storage. On the contrary, without dialysis to remove DMF, the as-synthesized nanoparticles sink rapidly in deionized water. These results suggest that DMF can inhibit BSA interaction with $\mathrm{NOBF}_{4} \cdot \mathrm{SPIONs} \mathrm{NOBF}_{4} \cdot \mathrm{SPION}$, having a high solubility in DMF, are inclined to huddle with DMF molecules even though BSA is present in the DMF/water solution. ${ }^{29}$ However, in the course of dialysis against water, the inhibition of DMF decreases along with DMF being diluted gradually in the samples. Therefore, hydrophilic BSA can be anchored onto the surfaces of SPIONs by means of its chemical groups with appreciable metal affinity, ${ }^{21}$ resulting in enhanced solubility of BSA.SPIONs in water.

Other water soluble compounds such as dopamine hydrochloride and PEG derivatives were used to stabilize SPIONs after the $\mathrm{NOBF}_{4}$ treatment using the same approach. However, these treatments result in serious SPIONs sediments in deionized water (Figure S3). It indicates that the molecular structures of these compounds play important roles in the surface functionalization of SPIONs. Compared with the low molecule compounds, the multiple anchoring 
sites on the BSA macromolecule enable high affinity with the surface metal atoms of SPIONs and therefore enhance the colloidal stability of BSA.SPIONs. ${ }^{21}$ The abundant amino/ carboxyl groups of BSA are favorable to post surface modifications with other functional materials such as antibody and fluorescent dye.

The BSA.SPIONs synthesized by this approach are monodisperse and uniform. The shape of the nanoparticles is maintained after surface modification (Figure 1A and B). No aggregation is observed, which is further confirmed by the DLS analysis (Figure 1C). As shown in the DLS data, the size of BSA.SPIONs is slightly larger than that observed from TEM. This is attributed to macromolecules being capped on SPIONs. The diameter of the as-synthesized hydrophobic SPIONs is about $8.0 \mathrm{~nm}$, and the BSA macromolecule in water is about $5.0 \mathrm{~nm}$ in size. ${ }^{37}$ Accordingly, the diameter of BSA.SPIONs is about $18.0 \mathrm{~nm}$ $(8.0+2 \times 5.0)$, coinciding with the hydrodynamic diameter analyzed by DLS.

The as-prepared BSA.SPIONs have excellent water solubility and colloidal stability (Figure 2). Figure 2A shows the colloidal stability of the BSA.SPIONs at various $\mathrm{pH}$ values. Over a wide range of $\mathrm{pH}$ values ( $\mathrm{pH} 4-13)$, the BSA.SPIONs solutions are optically clear without aggregations, except for the sample at $\mathrm{pH}$ 5.6. This is due to BSA.SPIONs being close to electroneutrality at $\mathrm{pH}$ 5.6, leading to a large number of precipitations (Figure 3C). BSA.SPIONs also exhibit good stability in sodium chloride water solutions with various ionic strengths (from $0.01 \mathrm{M}$ to $1 \mathrm{M}$, Figure $2 \mathrm{~B}$ ).

The phase transformation of SPIONs from organically soluble to water soluble was successfully achieved in this study. Instead of chloroform, the as-prepared BSA.SPIONs can only be solubilized in aqueous solution (Figure $2 \mathrm{C}$ ). The DLS data on the hydrodynamic diameters of BSA.SPIONs at diverse $\mathrm{pH}$ values and ionic strengths (Figure 3 ) also show its excellent colloidal stability.
As is well known, BSA is rich in the amino and carboxyl groups which can offer an effective cushioning effect in both acidic and alkaline solutions. Therefore, BSA surface functionalization enables the nanoparticles to be ionized at various $\mathrm{pH}$ values and resistant to harsh chemical surroundings. These experimental results ( $\mathrm{pH}$ approximately 7.4 and high ionic concentration) show favorable surface properties of BSA.SPIONs for intracellular and in vivo studies.

\section{In vitro relaxivity characterization of BSA.SPIONs}

SPIONs were synthesized for MRI for their known superparamagnetism. As can be seen in Figure 4A, all XRD diffraction peaks can be indexed to typical pattern of $\mathrm{Fe}_{3} \mathrm{O}_{4}$ (Joint Committee on Powder Diffraction Standards [JCPDS] 75-1609). Figure 4B shows the hysteresis loops of SPIONs at room temperature. The reversible hysteresis curves indicate the superparamagnetic nature of the nanoparticles.

The longitudinal $\left(\mathrm{T}_{1}\right)$ and transverse proton relaxation times $\left(\mathrm{T}_{2}\right)$ of BSA.SPION water solutions at different concentrations were measured as a function of $\mathrm{Fe}^{3+}$ concentration at $1.41 \mathrm{~T}, 37^{\circ} \mathrm{C}$. As shown in Figure $5 \mathrm{~A}$, the $\mathrm{r}_{2}$ value of the BSA.SPIONs was as high as $154.2 \mathrm{~s}^{-1}$ per $\mathrm{mM}$ of Fe ${ }^{3+}$. The $\mathrm{r}_{2} / \mathrm{r}_{1}$ value of 13.3 indicates significant advantage of BSA.SPIONs as negative MRI CAs. Only low dosage CA may be needed for imaging at high relaxivity. This is particularly useful for patients with weak organ functions. ${ }^{38}$ The $\mathrm{T}_{2}$-weighted MR images of BSA.SPIONs at various concentrations in Figure 5B give further evidence for favorable MRI application. MR signal intensities of the BSA.SPIONs water solutions are progressively reduced with decreasing $\mathrm{Fe}^{3+}$ concentration.

It is well known that the surface of nanoparticles is capped by biomolecules (proteins, sugars, and lipids) upon coming into contact with biological systems, resulting in the formation of a protein "corona" that is strongly associated with the nanoparticle surface. ${ }^{39}$ Therefore, the MR relaxivity of nanoparticles could be affected by this protein "corona". ${ }^{40}$ In this study,
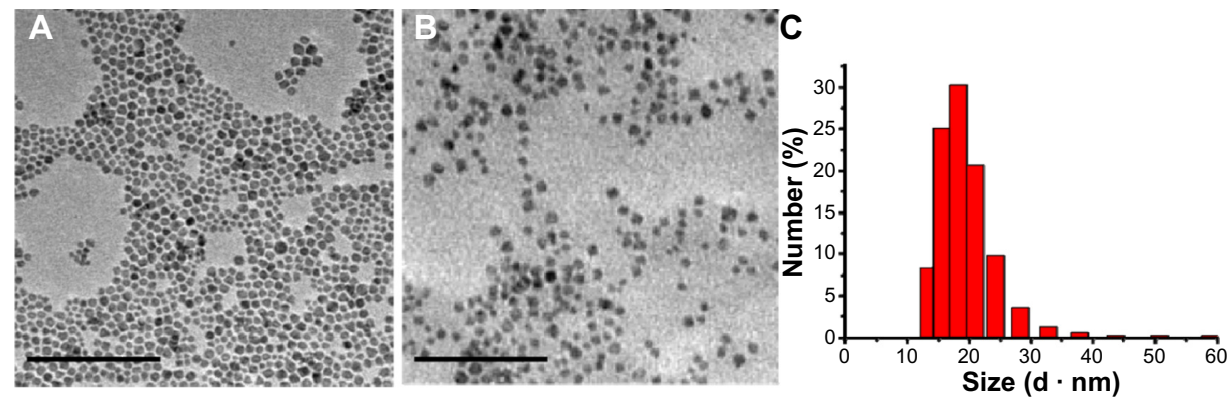

Figure I Transmission electron microscopy images of hydrophobic superparamagnetic iron oxide nanoparticles.

Notes: (A) Superparamagnetic iron oxide nanoparticles (SPIONs) in chloroform and (B) bovine serum albumin surface functionalized hydrophobic SPIONs (BSA.SPIONs) in borate buffer without size selection. (C) Dynamic light scattering data of the BSA.SPIONs in borate buffer. Scale bar is $100 \mathrm{~nm}$. 

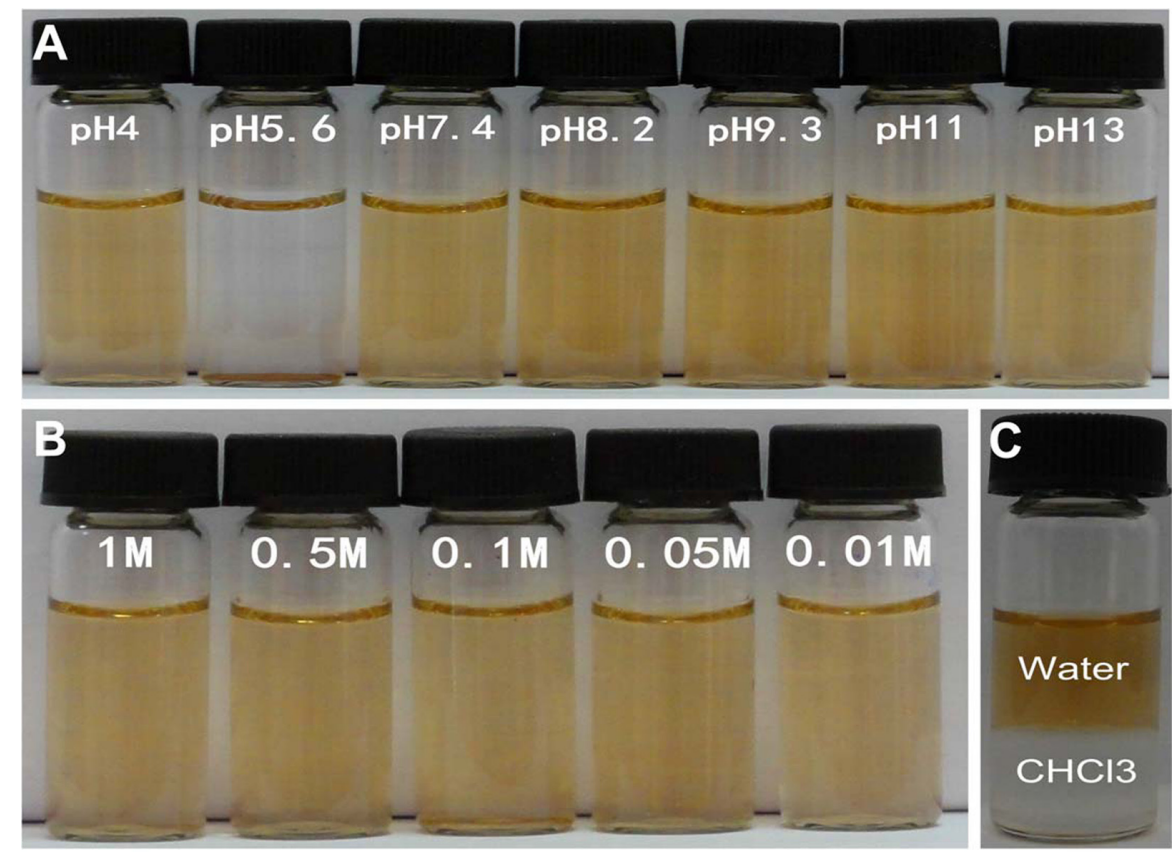

Figure 2 Digital images of the bovine serum albumin surface functionalized hydrophobic superparamagnetic iron oxide nanoparticles in corresponding solutions with different $\mathrm{pH}$ values.

Notes: (A) Bovine serum albumin surface functionalized hydrophobic superparamagnetic iron oxide nanoparticles (BSA.SPIONs) corresponding solutions with different pH values and (B) with various ionic strengths in sodium chloride water solutions with (C) demonstration of successful phase transfer of the BSA.SPIONs. All samples before optical photography were placed at room temperature for 7 days of storage.

A

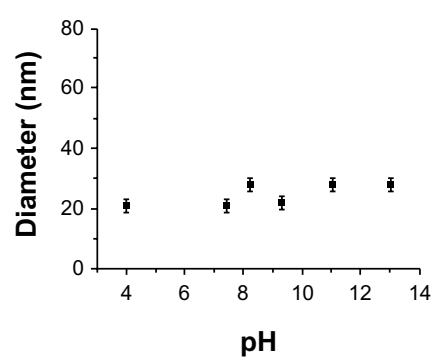

B

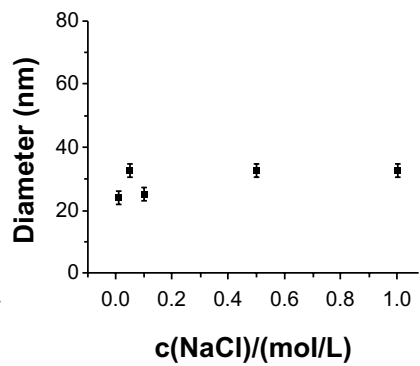

C

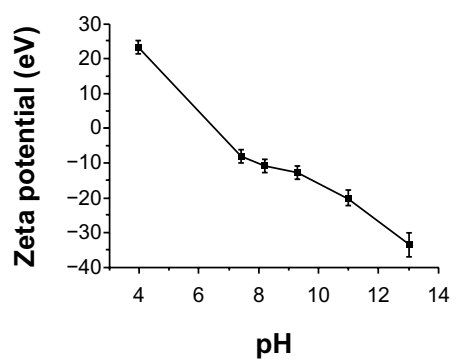

Figure 3 The hydrodynamic diameters of the bovine serum albumin surface functionalized hydrophobic superparamagnetic iron oxide nanoparticles.

Notes: (A) Bovine serum albumin surface functionalized hydrophobic superparamagnetic iron oxide nanoparticles (BSA.SPIONs) at various pH buffer solutions and (B) in sodium chloride water solutions with different ionic strengths analyzed by dynamic light scattering after 7 days of storage at room temperature. (C) The zeta potentials of BSA.SPIONs at various $\mathrm{pH}$ buffer solutions.

Abbreviation: $\mathrm{NaCl}$, sodium chloride.
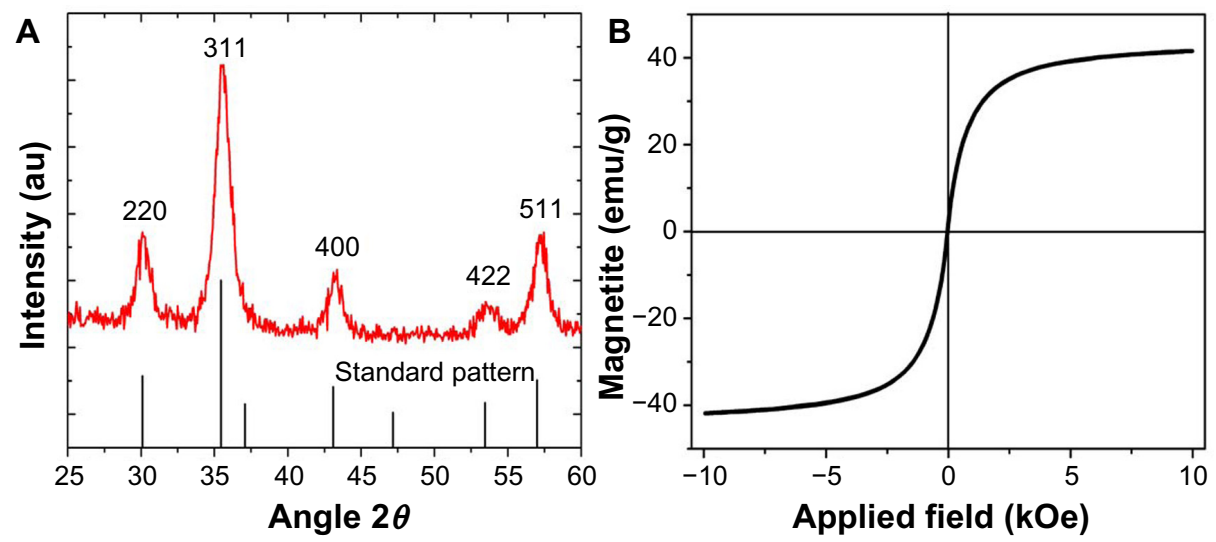

Figure 4 Analysis of superparamagnetic iron oxide nanoparticles.

Notes: X-ray diffraction pattern $(\mathbf{A})$ and magnetic hysteresis curve $(\mathbf{B})$ of the hydrophobic superparamagnetic iron oxide nanoparticles. 
A

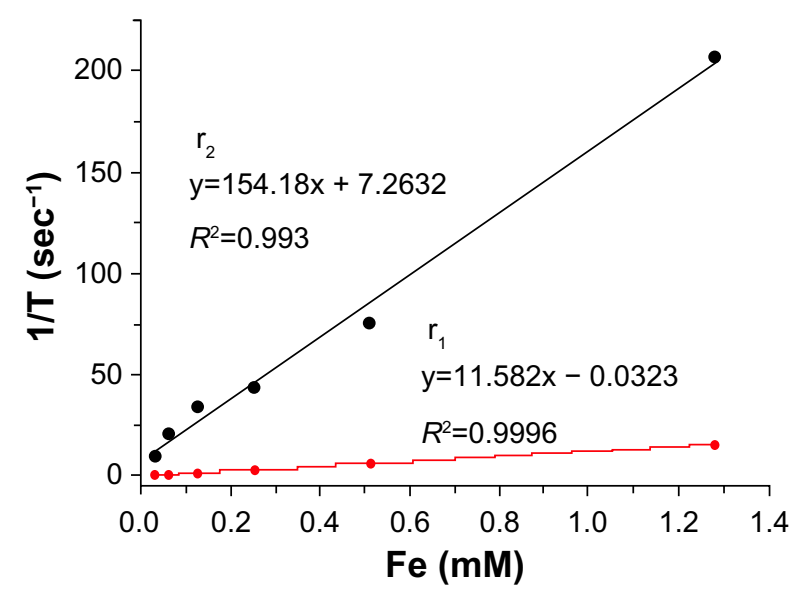

B

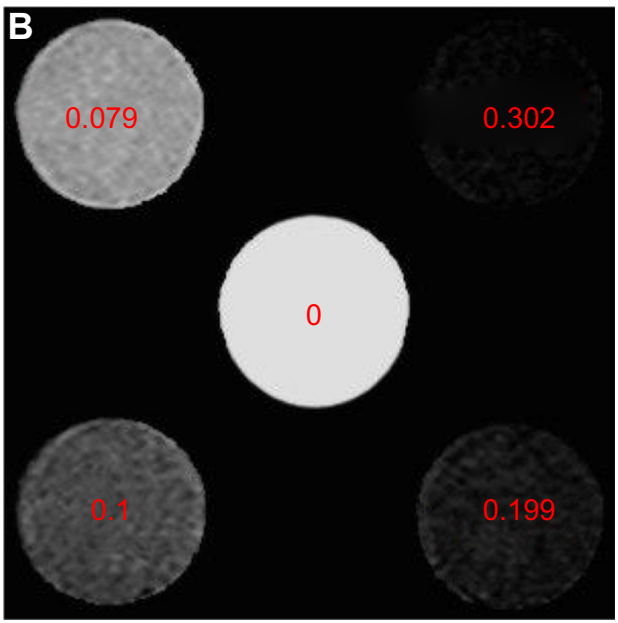

Figure 5 Relaxivity curves and magnetic resonance images of BSA.SPIONs.

Notes: (A) The $r_{1}$ and $r_{2}$ relaxivity curves obtained from water solutions of bovine serum albumin surface functionalized hydrophobic superparamagnetic iron oxide nanoparticles (BSA.SPIONs). (B) $\mathrm{T}_{2}$-weighted magnetic resonance images of BSA.SPIONs with various concentrations (from 0.079 to $0.302 \mathrm{mM}$ Fe ${ }^{3+}$ ).
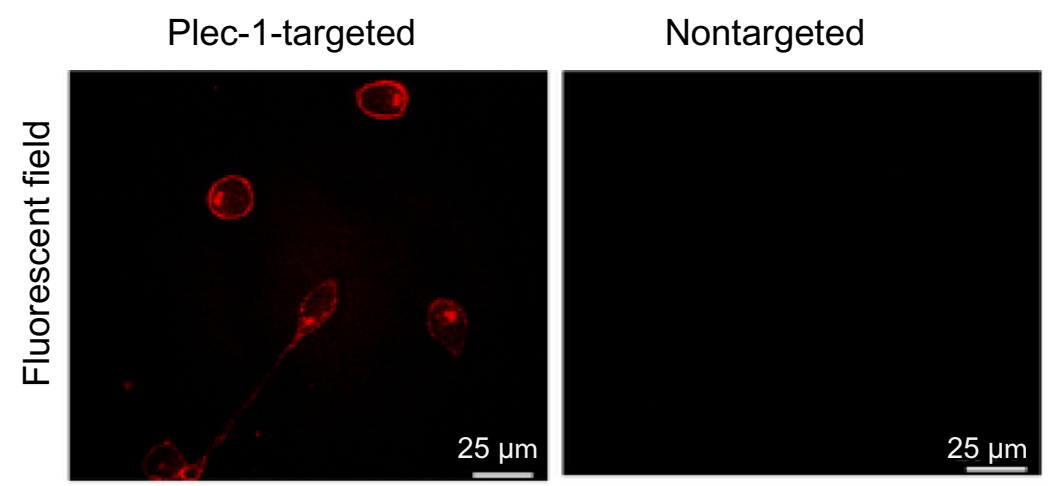

\section{Plec-1-targeted + block}
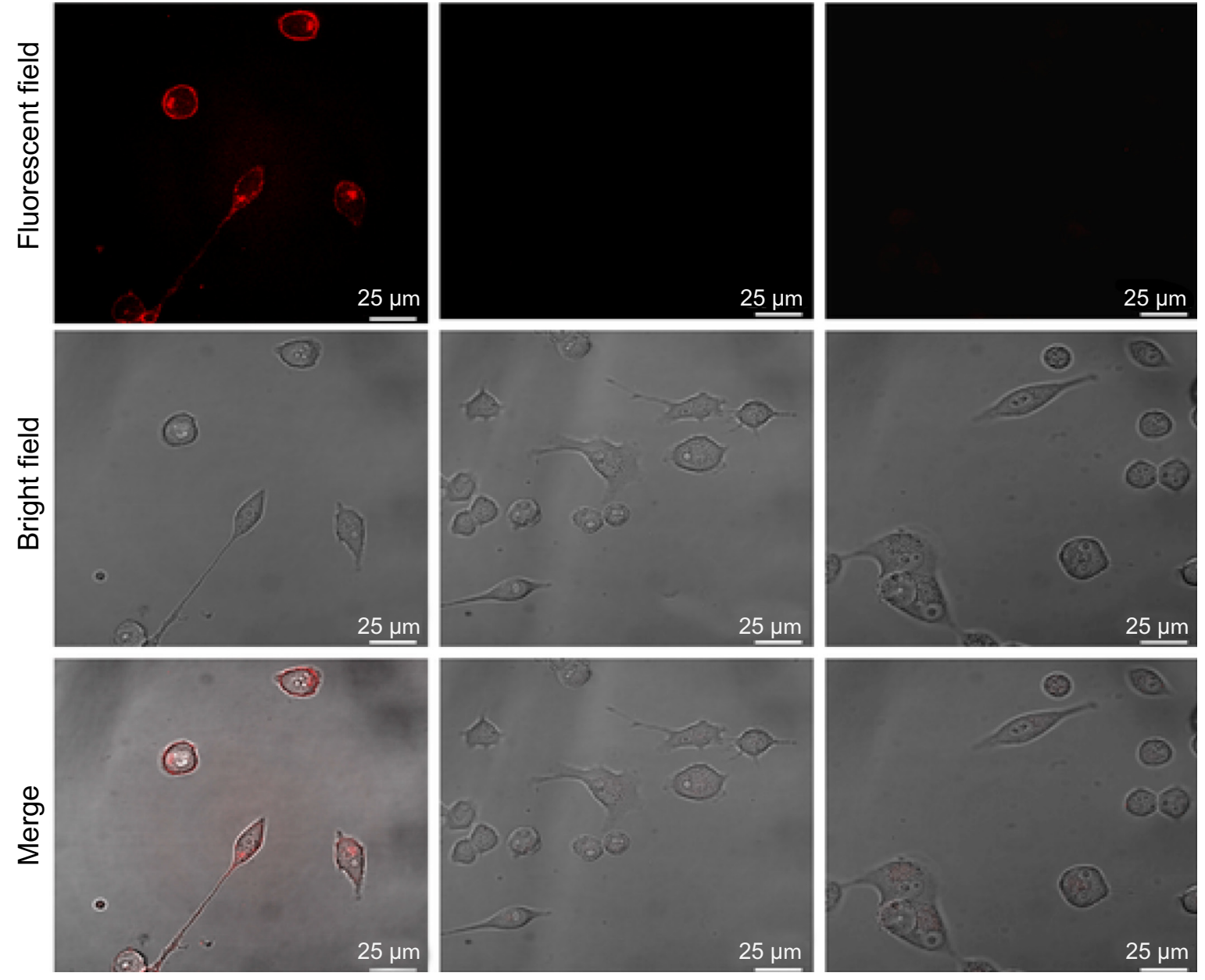

Figure 6 Targeted imaging of Panc-I cells with Plec-I-targeted dyeBSA.SPIONs-mAb bioconjugates.

Notes: Panc-I cells (Plec-I-positive) were incubated with Plec-I-targeted and nontargeted bovine serum albumin surface functionalized hydrophobic superparamagnetic iron oxide nanoparticles (BSA.SPIONs) for 5 minutes. To further determine targeting specificity, cells were preincubated with free Plec-I antibodies before adding Plec-I-targeted dye BSA.SPIONs-mAb bioconjugates. 
BSA.SPIONs are precoated with BSA protein, which have antifouling properties and repressing the "corona" capability in a biological environment. It was found that BSA.SPIONs are suitable for MRI applications based on their relaxivity data.

\section{In vitro laser scanning confocal and $M R$ imaging}

Successful conjugation of Cy5 dyes and anti-Plec-1 antibodies to BSA.SPIONs are demonstrated (Figure S4A) for targeted optical and MR imaging. As shown in the fluorescent spectra

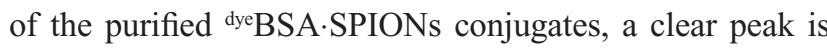
observed at $658 \mathrm{~nm}$ with $630 \mathrm{~nm}$ excitation. This is consistent with the fluorescent spectra of the free Cy 5 dye, indicating that the Cy5 dyes are successfully conjugated to the BSA.SPIONs nanoparticles. To verify the conjugation of anti-Plec-1 antibodies to ${ }^{\text {dye } B S A}$.SPIONs, a test strip technology was used for the secondary antibodies to recognize the primary antibodies on nanoparticles. ${ }^{30}$ As can be seen in Figure S4B, there is a distinguishable narrow and yellow band on the test strip for the ${ }^{d y}$ BSA.SPIONs-mAb bioconjugate, but no signal is observed for the nontargeted dye BSA.SPIONs conjugate. It is concluded, therefore, the anti-Plec-1 primary antibodies were successfully conjugated to the ${ }^{\text {dye }}$ BSA.SPIONs nanoparticles.

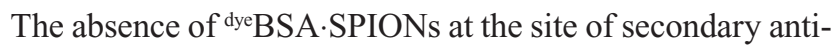
bodies line shows their potent antifouling capability, which is favorable for the following cell targeted imaging.
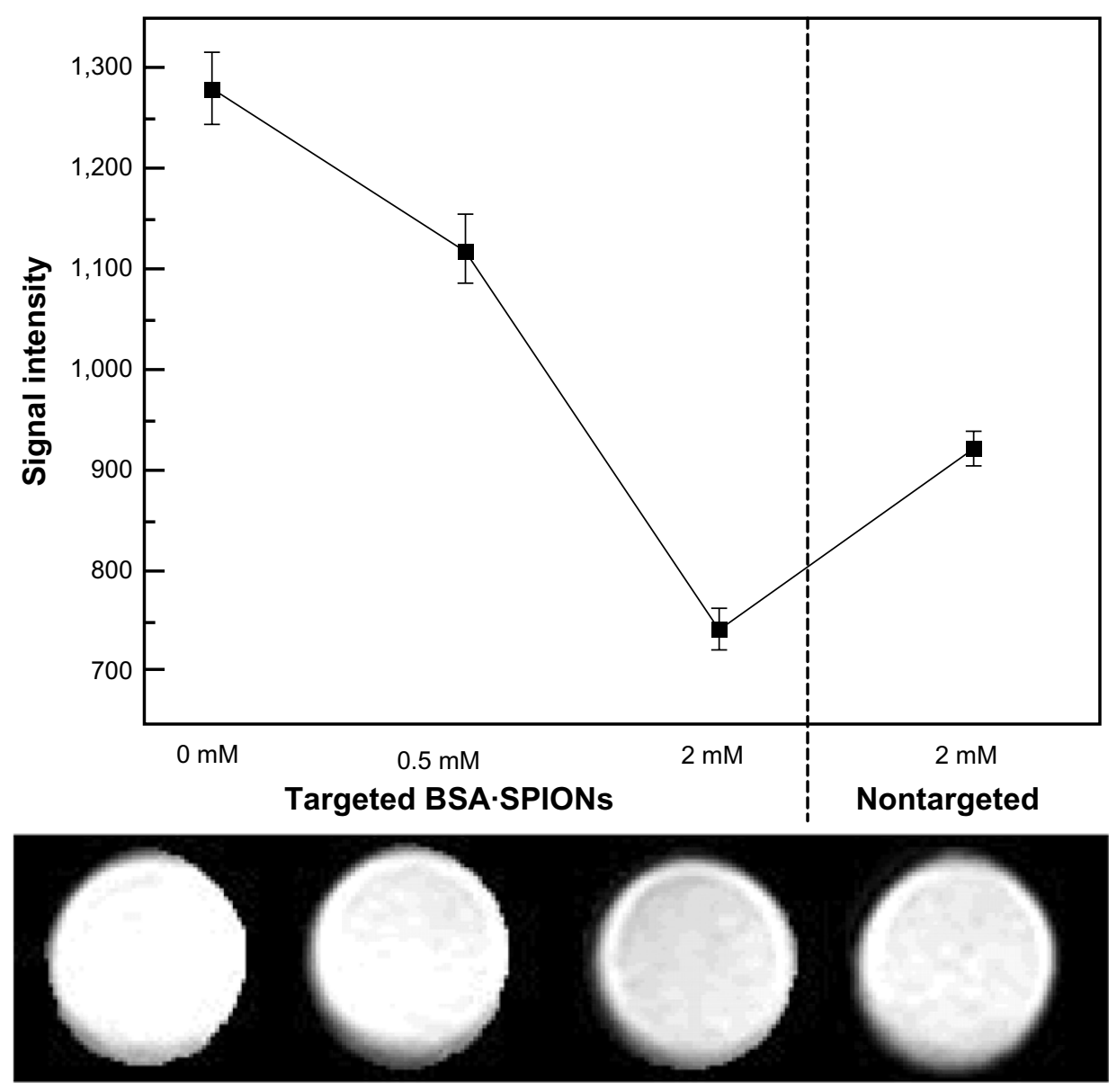

$0 \mathrm{mM}$

$0.5 \mathrm{mM}$

$2 \mathrm{mM}$

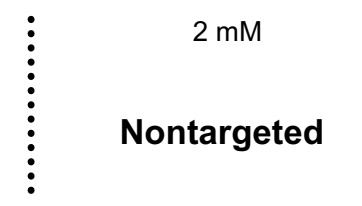

Figure 7 Magnetic resonance images of Panc-I cells

Notes: Signal intensity curve (top) and $\mathrm{T}_{2}$-weighted (bottom) magnetic resonance imaging of Panc-I cells incubated with Plec-I-targeted dyebovine serum albumin surface functionalized hydrophobic superparamagnetic iron oxide nanoparticles-monoclonal antibody bioconjugates ( 0 to $2 \mathrm{mM})$ and nontargeted dyebovine serum albumin surface functionalized hydrophobic superparamagnetic iron oxide nanoparticles conjugates ( $2 \mathrm{mM})$ for 2 hours.

Abbreviation: BSA.SPIONs, bovine serum albumin surface functionalized hydrophobic superparamagnetic iron oxide nanoparticles. 
Figure 6 shows the targeting capability of Plec-1-targeted ${ }^{\text {dye }}$ BSA.SPIONs-mAb bioconjugates to Panc-1 cells (Plec-1 overexpressed). ${ }^{27}$ The laser scanning confocal images show

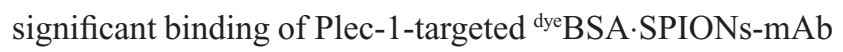
to the Panc-1 cells but not the nontargeted dye BSA.SPIONs. This targeting specificity is further confirmed by competitive inhibition of the fluorescence cell assay. After blocking with free anti-Plec-1 antibodies, the binding of ${ }^{d y}$ BSA.SPIONs-mAb to the Panc-1 cells (Figure 6C) is significantly inhibited.

Nonspecific cellular binding of nanoparticles has been a complex issue. It is associated with hydrophobic interactions between the ligands of nanoparticles and lipids on the cell membranes. It can also be attributed to the electrostatic interactions between the cells and the charged nanoparticle surface functional groups. ${ }^{13,16,17}$ BSA has been widely used for nonspecific binding reduction in biological detection..$^{21,41,42}$ In this study, the BSA surface tailored SPIONs was shown to exhibit superior antifouling on reduction of nonspecific cellular binding based on the cell assay results (Figure 6). BSA has been found in this study to serve two major functionalities, namely the ligand exchange for water solubilization of SPIONs and antifouling for reduction of nonspecific cellular binding.

The BSA protein surface engineered SPIONs can repress protein corona upon contact in the biological system. It is reported that protein corona can significantly reduce active targeting yield, because protein corona induced screening of nanoparticle targeting ligands. ${ }^{43,44} \mathrm{In}$ this study, BSA protein capped on SPIONs has the capability to reduce protein corona. Thus, the antibodies linked on the BSA are fully exposed to their targets on a separate surface. Also, cellular immunofluorescence assays have shown that they are targeting and are effective.

Panc-1 cell MR scanning was performed after incubation with Plec-1-targeted dyeBSA.SPIONs-mAb and nontargeted ${ }^{d y e} B S A \cdot S P I O N s$ conjugates, respectively. As shown in Figure 7, Panc-1 cells incubated with Plec-1-targeted ${ }^{d y e} B S A \cdot S P I O N s-m A b$ exhibit significant $T_{2}$ reduction when compared with nontargeted dye BSA.SPIONs at the same concentration. The incubation time for MR scanning is 2 hours, which is much longer than that for fluorescent imaging (5 minutes). The samples after 5 minutes of incubation show no signal changes in the MRI images due to its lower sensitivity compared to optical imaging. By extending incubation time, cellular internalization of nanoparticles results in nonspecific signal enhancement for nontargeted dyeBSA.SPIONs conjugates in both MR and fluorescent imaging (Figure S5). It is worth noting that the Plec-1-targeted dyeBSA.SPIONs$\mathrm{mAb}$ bioconjugates are mainly located on the cell membrane with few nanoparticles internalized into cytoplasm, while the nontargeted dye BSA.SPIONs predominately internalized into the cytoplasm of Panc-1 cells (Figure S5). This difference is

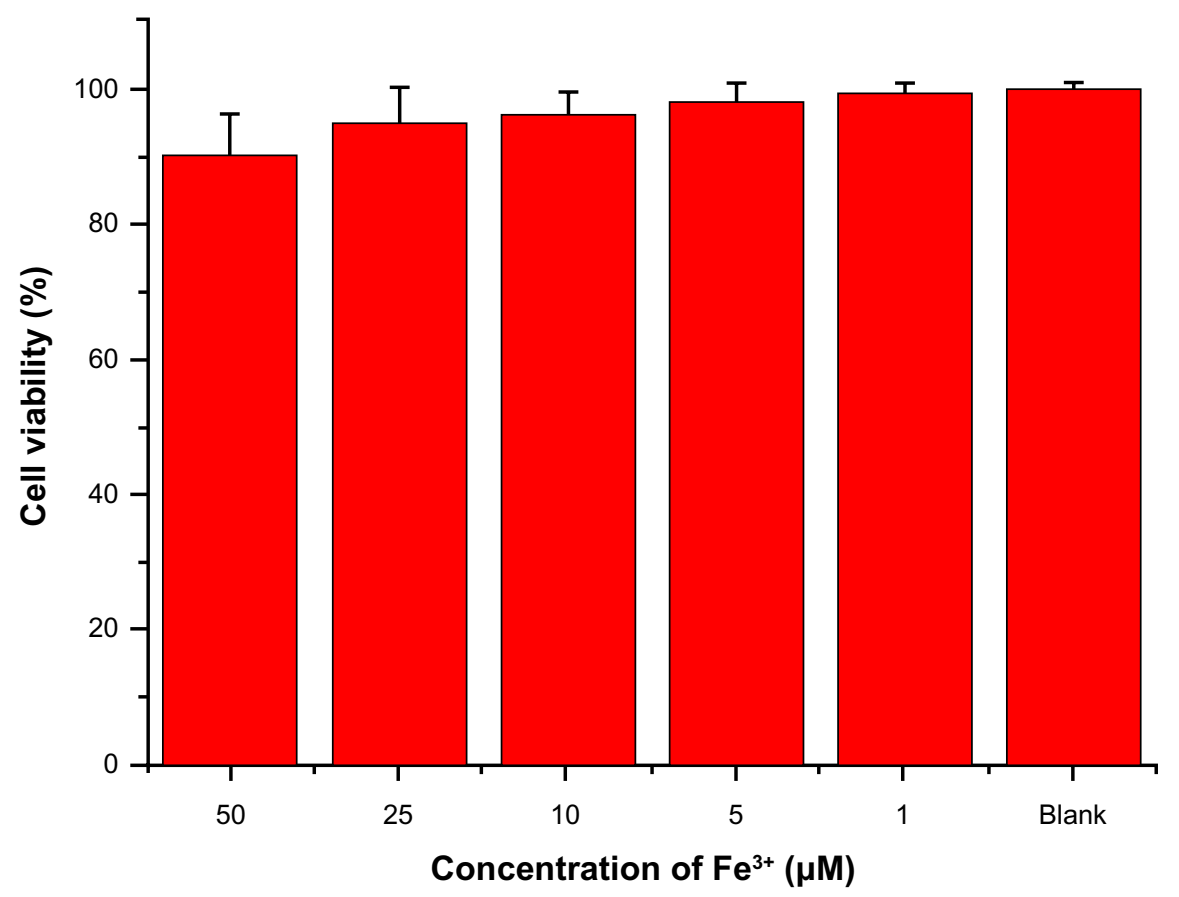

Figure 8 Viability of L02 cells incubated with bovine serum albumin surface functionalized hydrophobic superparamagnetic iron oxide nanoparticles for 24 hours was measured using the 3-(4, 5-dimethylthiazol-2-yl)-2, 5-diphenyl-tetrazolium bromide assay.

Note: Viability measurements were normalized to cells grown in the absence of any nanoparticles. 
attributable to the validity of the antibody-mediated targeting. The combination of optical and MR imaging is clinically advantageous, as shown in this study, as MRI provides anatomical investigation in vivo while high sensitivity is ensured by optical imaging.

This study reports a novel nanoparticle surface engineering approach. Upon conjugation with antibodies, the prepared nanoprobe has good cell targeted imaging capability. Also, their biodistribution, an in vivo imaging study, and an in vivo toxicity study will be considered in the next research plan in our laboratory.

\section{Cytotoxicity study}

In vitro cytotoxicity of BSA.SPIONs was evaluated against the normal L02 cell line using the MTT assay (Figure 8). After 24 hours incubation with BSA.SPIONs at various $\mathrm{Fe}^{3+}$ concentrations, the viability of L02 cells is over $80 \%$, even at $50 \mu \mathrm{M}$ of $\mathrm{Fe}^{3+}$. It indicates hypotoxicity of BSA.SPIONs against normal L02 cells, which has great potential for in vivo imaging.

\section{Conclusion}

A novel and facile approach was developed for surface functionalization of SPIONs with BSA biomacromolecule. BSA.SPIONs have been found to exhibit excellent biocompatibility, colloidal stability, and high relaxivity. Nonspecific cellular binding has been effectively reduced by this new approach. Excellent performance of dye BSA.SPIONs-mAb bioconjugates are demonstrated in the bimodal imaging of pancreatic cancer cells. Several major advantages of the ${ }^{\text {dye}}$ BSA.SPIONs-mAb bioconjugates, including simple synthesis, good biocompatibility and stability, high relaxivity, and targeted imaging, make these nanosystems ideal candidates as imaging $\mathrm{CA}$ and will show promise in early cancer diagnosis.

\section{Acknowledgment}

This work was partially supported by the National Natural Science Foundation of China (81371618, 81171393, and 81271629), the Key Basic Research Project of Shanghai Science and Technology (10JC1412900), Shanghai Innovation program (14ZZ039), and the Nanotechnology Program of Shanghai Science and Technology Committee (11nm0504500 and 12nm0500800).

\section{Supporting information available}

Fourier transform infrared spectra, digital images of superparamagnetic iron oxide nanoparticles, fluorescent spectra of free $\mathrm{Cy} 5$ dye and dyebovine serum albumin surface functionalized hydrophobic superparamagnetic iron oxide nanoparticles, and confocal images are available free of charge via the Internet.

\section{Disclosure}

The authors report no conflicts of interest in this work.

\section{References}

1. Schneider G, Siveke JT, Eckel F, Schmid RM. Pancreatic cancer: basic and clinical aspects. Gastroenterology. 2005;128(6):1606-1625.

2. Jemal A, Siegel R, Ward E, Hao Y, Xu J, Thun MJ. Cancer statistics, 2009. CA Cancer J Clin. 2009;59(4):225-249.

3. Foy SP, Manthe RL, Foy ST, Dimitrijevic S, Krishnamurthy N, Labhasetwar V. Optical imaging and magnetic field targeting of magnetic nanoparticles in tumors. ACS Nano. 2010;4(9):5217-5224.

4. Wang QB, Han Y, Jiang TT, et al. MR Imaging of activated hepatic stellate cells in liver injured by $\mathrm{CCl} 4$ of rats with integrin-targeted ultrasmall superparamagnetic iron oxide. Eur Radiol. 2011;21(5): 1016-1025.

5. Liu F, Laurent S, Fattahi H, Vander Elst L, Muller RN. Superparamagnetic nanosystems based on iron oxide nanoparticles for biomedical imaging. Nanomedicine (Lond). 2011;6(3):519-528.

6. Cheng FY, Su CH, Yang YS, et al. Characterization of aqueous dispersions of $\mathrm{Fe} 3 \mathrm{O} 4$ nanoparticles and their biomedical applications. Biomaterials. 2005;26(7):729-738.

7. Thorek DL, Chen AK, Czupryna J, Tsourkas A. Superparamagnetic iron oxide nanoparticle probes for molecular imaging. Ann Biomed Eng. 2006;34(1):23-38.

8. Bulte JW, Kraitchman DL. Iron oxide MR contrast agents for molecular and cellular imaging. NMR Biomed. 2004;17(7):484-499.

9. Sun S, Zeng H, Robinson DB, et al. Monodisperse MFe2O4 (M = Fe, Co, Mn) nanoparticles. J Am Chem Soc. 2004;126(1):273-279.

10. Yu WW, Chang E, Falkner JC, et al. Forming biocompatible and nonaggregated nanocrystals in water using amphiphilic polymers. J Am Chem Soc. 2007;129(10):2871-2879.

11. $\mathrm{Xu} \mathrm{C}, \mathrm{Xu} \mathrm{K}, \mathrm{Gu} \mathrm{H}$, et al. Dopamine as a robust anchor to immobilize functional molecules on the iron oxide shell of magnetic nanoparticles. J Am Chem Soc. 2004;126(32):9938-9939.

12. Niu D, Li Y, Qiao X, et al. A facile approach to fabricate functionalized superparamagnetic copolymer-silica nanocomposite spheres. Chem Commun (Camb). 2008;(37):4463-4465.

13. Chen H, Wang L, Yeh J, et al. Reducing non-specific binding and uptake of nanoparticles and improving cell targeting with an antifouling PEO-b-PgammaMPS copolymer coating. Biomaterials. 2010;31(20): 5397-5407.

14. Salvador-Morales C, Zhang L, Langer R, Farokhzad OC. Immunocompatibility properties of lipid-polymer hybrid nanoparticles with heterogeneous surface functional groups. Biomaterials. 2009;30(12):2231-2240.

15. Cho EC, Xie J, Wurm PA, Xia Y. Understanding the role of surface charges in cellular adsorption versus internalization by selectively removing gold nanoparticles on the cell surface with a I2/KI etchant. Nano Lett. 2009;9(3):1080-1084.

16. Kairdolf BA, Mancini MC, Smith AM, Nie S. Minimizing nonspecific cellular binding of quantum dots with hydroxyl-derivatized surface coatings. Anal Chem. 2008;80(8):3029-3034.

17. Bentzen EL, Tomlinson ID, Mason J, et al. Surface modification to reduce nonspecific binding of quantum dots in live cell assays. Bioconjug Chem. 2005;16(6):1488-1494.

18. Simpson CA, Agrawal AC, Balinski A, Harkness KM, Cliffel DE. Short-chain PEG mixed monolayer protected gold clusters increase clearance and red blood cell counts. ACS Nano. 2011;5(5): $3577-3584$. 
19. Strable E, Bulte JWM, Moskowitz B, Vivekanandan K, Allen M, Douglas T. Synthesis and characterization of soluble iron oxidedendrimer composites. Chem Mater. 2001;13(6):2201-2209.

20. Wakayama J, Sekiguchi H, Akanuma S, Ohtani T, Sugiyama S. Methods for reducing nonspecific interaction in antibody-antigen assay via atomic force microscopy. Anal Biochem. 2008;380(1):51-58.

21. Zhang B, Wang X, Liu F, Cheng Y, Shi D. Effective reduction of nonspecific binding by surface engineering of quantum dots with bovine serum albumin for cell-targeted imaging. Langmuir. 2012;28(48): 16605-16613.

22. Zhang B, Li Q, Yin P, et al. Ultrasound-triggered BSA/SPION hybrid nanoclusters for liver-specific magnetic resonance imaging. ACS Appli Mater Interfaces. 2012;4(12):6479-6486.

23. Remsen LG, McCormick CI, Roman-Goldstein S, et al. MR of carcinoma-specific monoclonal antibody conjugated to monocrystalline iron oxide nanoparticles: the potential for noninvasive diagnosis. AJNR Am J Neuroradiol. 1996;17(3):411-418.

24. Huang J, Wang L, Lin R, et al. Casein-coated iron oxide nanoparticles for high MRI contrast enhancement and efficient cell targeting. ACS Appl Mater Interfaces. 2013;5(11):4632-4639.

25. Huang J, Xie J, Chen K, et al. HSA coated MnO nanoparticles with prominent MRI contrast for tumor imaging. Chem Commun (Camb). 2010;46(36):6684-6686.

26. Lee JH, Lee K, Moon SH, Lee Y, Park TG, Cheon J. All-in-one target-cell-specific magnetic nanoparticles for simultaneous molecular imaging and siRNA delivery. Angew Chem Int Ed Engl. 2009;48(23): 4174-4179.

27. Bausch D, Thomas S, Mino-Kenudson M, et al. Plectin-1 as a novel biomarker for pancreatic cancer. Clin Cancer Res. 2011;17(2):302-309.

28. Kelly KA, Bardeesy N, Anbazhagan R, et al. Targeted nanoparticles for imaging incipient pancreatic ductal adenocarcinoma. PLoS Med. 2008;5(4):e85.

29. Dong A, Ye X, Chen J, et al. A generalized ligand-exchange strategy enabling sequential surface functionalization of colloidal nanocrystals. J Am Chem Soc. 2011;133(4):998-1006.

30. Yang Q, Gong X, Song T, et al. Quantum dot-based immunochromatography test strip for rapid, quantitative and sensitive detection of alpha fetoprotein. Biosens Bioelectron. 2011;30(1):145-150.

31. Mahmoudi M, Simchi A, Imani M, et al. A new approach for the in vitro identification of the cytotoxicity of superparamagnetic iron oxide nanoparticles. Colloids Surf B Biointerfaces. 2010;75(1):300-309.
32. Carter DC, Ho JX. Structure of serum albumin. Adv Prot Chem. 1994;45:153-203.

33. Huang X, Li BY, Zhang H, Hussain I, Liang LY, Tan BE. Facile preparation of size-controlled gold nanoparticles using versatile and end-functionalized thioether polymer ligands. Nanoscale. 2011;3(4): 1600-1607.

34. Cui Y, Gong XQ, Zhu SJ, et al. An effective modified method to prepare highly luminescent, highly stable water-soluble quantum dots and its preliminary application in immunoassay. J Mater Chem. 2012;22(2): 462-469.

35. Tang ZH, Xu B, Wu BH, Germann MW, Wang GL. Synthesis and structural determination of multidentate 2,3-dithiol-stabilized $\mathrm{Au}$ clusters. J Am Chem Soc. 2010;132(10):3367-3374.

36. Lutz HD, Himmrich J, Schmidt M. Lattice vibration spectra. Part LXXXVI. Infrared and Raman spectra of baryte-type T1ClO4, TlBF4, and NH4BF4 single crystals and of 11B-enriched NH4BF4. J Alloys Compd. 1996;241(1-2):1-9.

37. Wright AK, Thompson MR. Hydrodynamic structure of bovine serum albumin determined by transient electric birefringence. Biophys $J$. 1975;15(2 Pt 1):137-141.

38. Burtea C, Laurent S, Murariu O, et al. Molecular imaging of alpha v beta3 integrin expression in atherosclerotic plaques with a mimetic of RGD peptide grafted to Gd-DTPA. Cardiovasc Res. 2008;78(1):148-157.

39. Monopoli MP, Walczyk D, Campbell A, et al. Physical-chemical aspects of protein corona: relevance to in vitro and in vivo biological impacts of nanoparticles. J Am Chem Soc. 2011;133(8):2525-2534.

40. Amiri H, Bordonali L, Lascialfari A, et al. Protein corona affects the relaxivity and MRI contrast efficiency of magnetic nanoparticles. Nanoscale. 2013;5(18):8656-8665.

41. Sakai G, Nakata S, Uda T, Miura N, Yamazoe N. Highly selective and sensitive SPR immunosensor for detection of methamphetamine. Electrochim Acta. 1999;44(21-22):3849-3854.

42. Festag G, Steinbruck A, Wolff A, Csaki A, Moller R, Fritzsche W. Optimization of gold nanoparticle-based DNA detection for microarrays. J Fluoresc. 2005;15(2):161-170.

43. Mirshafiee V, Mahmoudi M, Lou KY, Cheng JJ, Kraft ML. Protein corona significantly reduces active targeting yield. Chem Commun (Camb). 2013;49(25):2557-2559.

44. Salvati A, Pitek AS, Monopoli MP, et al. Transferrin-functionalized nanoparticles lose their targeting capabilities when a biomolecule corona adsorbs on the surface. Nat Nanotechnol. 2013;8(2):137-143. 


\section{Supplementary materials}

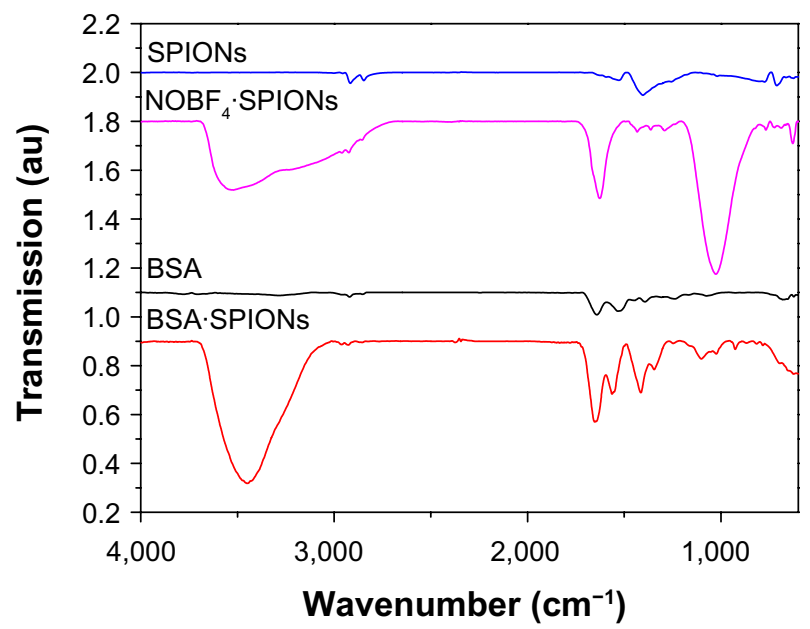

Figure SI Fourier transform infrared spectra of the original superparamagnetic iron oxide nanoparticles, tetrafluoroborate superparamagnetic iron oxide nanoparticles, bovine serum albumin, and bovine serum albumin surface functionalized hydrophobic superparamagnetic iron oxide nanoparticles.

Abbreviations: BSA, bovine serum albumin; BSA.SPIONs, bovine serum albumin surface functionalized hydrophobic superparamagnetic iron oxide nanoparticles; $\mathrm{NOBF}_{4}$.SPIONS; tetrafluoroborate superparamagnetic iron oxide nanoparticles; SPIONS, superparamagnetic iron oxide nanoparticles.

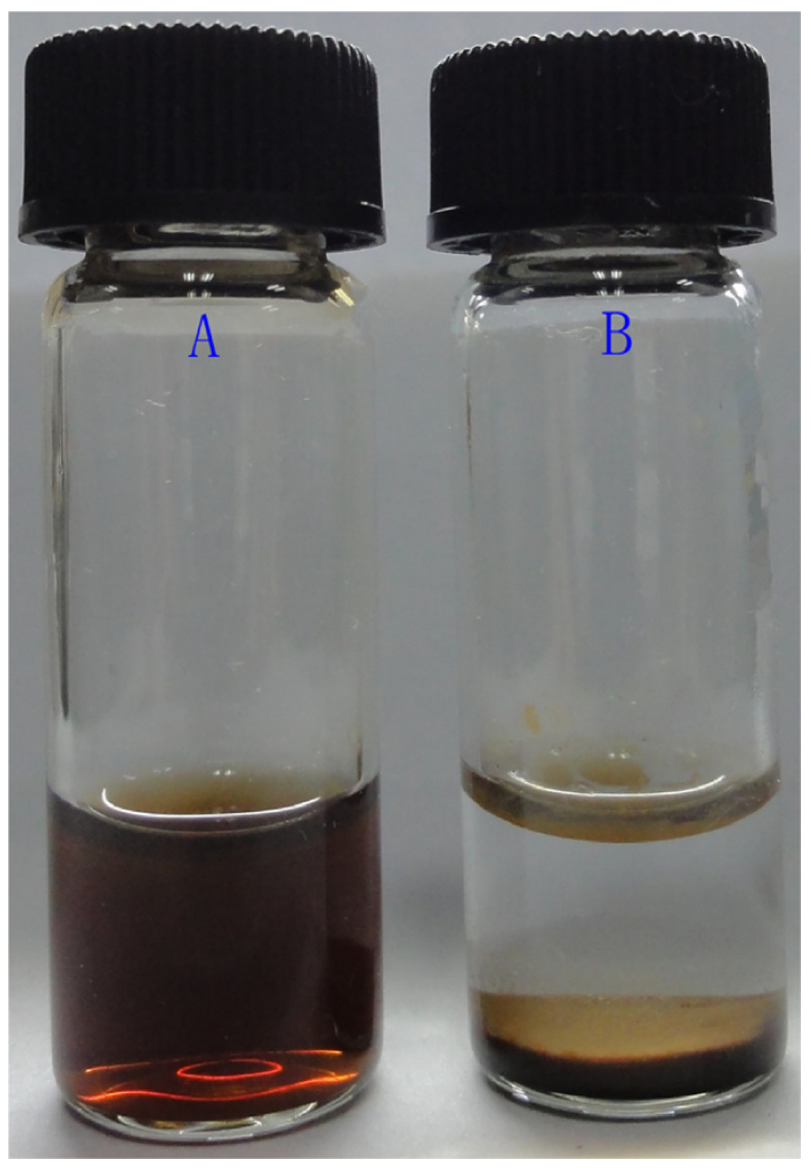

Figure S2 Optical photographs of bovine serum albumin surface functionalized hydrophobic superparamagnetic iron oxide nanoparticles showing the necessity of dialysis to remove the dimethylformamide.

Notes: The dispersion of dialyzed bovine serum albumin surface functionalized hydrophobic superparamagnetic iron oxide nanoparticles is optically clear (A) while the sample without dialysis has obvious sediments (B). 


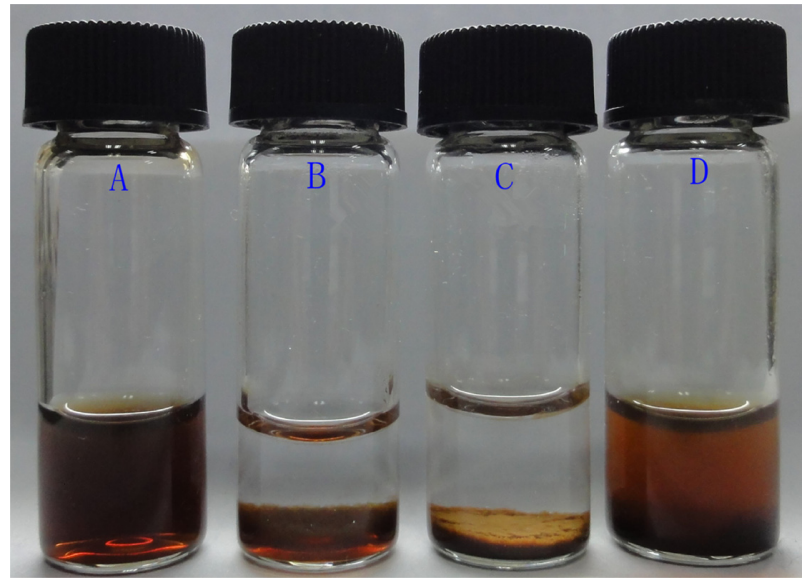

Figure S3 Digital images of superparamagnetic iron oxide nanoparticles.

Notes: Superparamagnetic iron oxide nanoparticles redispersed in deionized water and functionalized by various water soluble polymers including (A) bovine serum albumin, (B) dopamine hydrochloride, (C) $\mathrm{NH}$ 2-polyethylene glycol-COOH (molecular weight of $2 \mathrm{kDa}$ ), and (D) $\mathrm{NH}$ 2-polyethylene glycol-SH (molecular weight of 3.5 kDa) after 7 days of storage at room temperature.
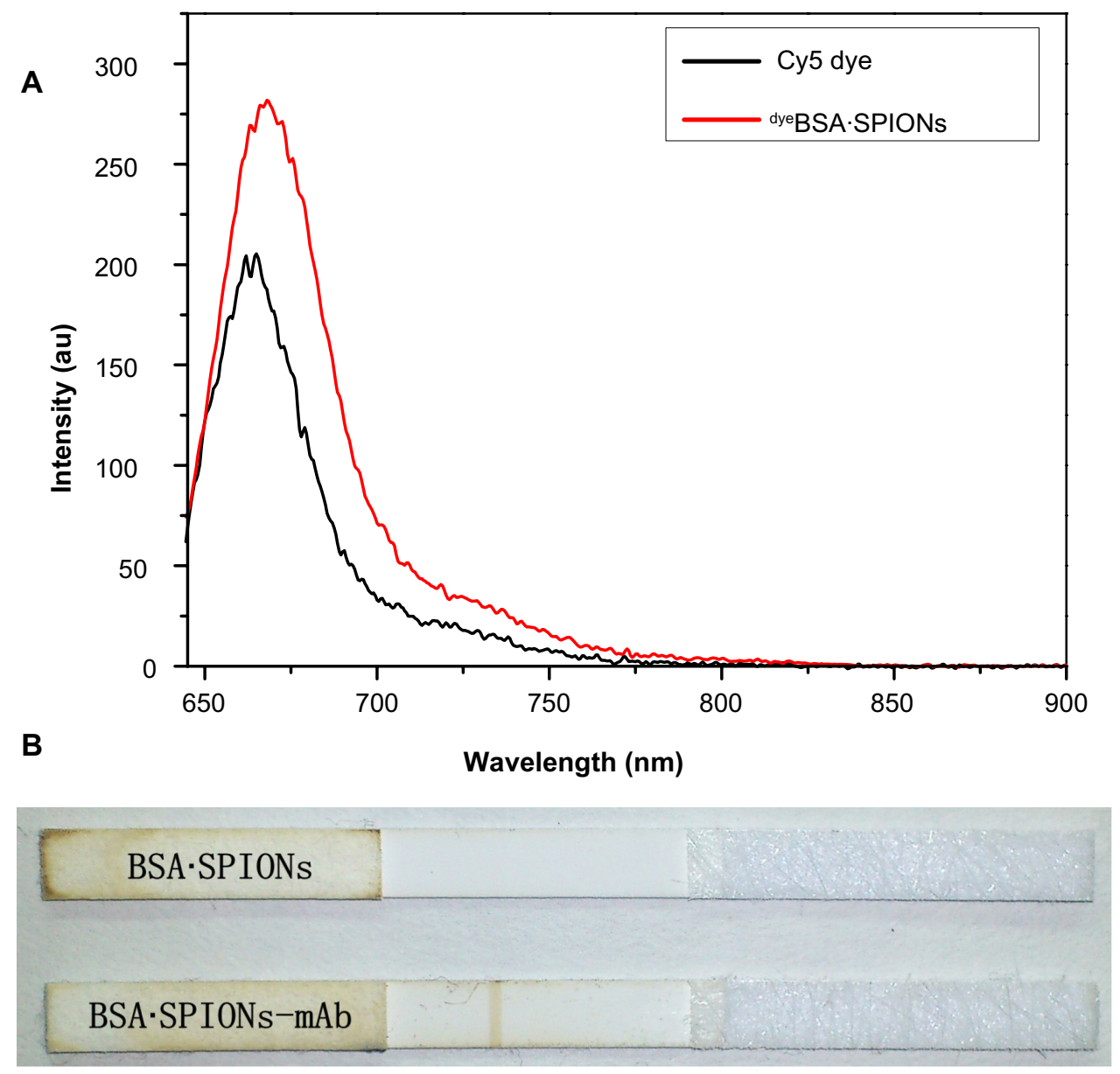

Figure S4 Fluorescence spectra and test strips of functionalized hydrophobic superparamagnetic iron oxide nanoparticles.

Notes: (A) Fluorescence spectra of free Cy5 dye and dyebovine serum albumin surface functionalized hydrophobic superparamagnetic iron oxide nanoparticles (dyeBSA.SPIONs). (B) Digital images of test trips using dyeBSA.SPIONs and dyeBSA.SPIONs-monoclonal antibody bioconjugates.

Abbreviations: dyeBSA.SPIONs, dyebovine serum albumin surface functionalized hydrophobic superparamagnetic iron oxide nanoparticles; dyeBSA.SPIONs-mAb, dyeBSA.SPIONs-monoclonal antibody bioconjugates. 

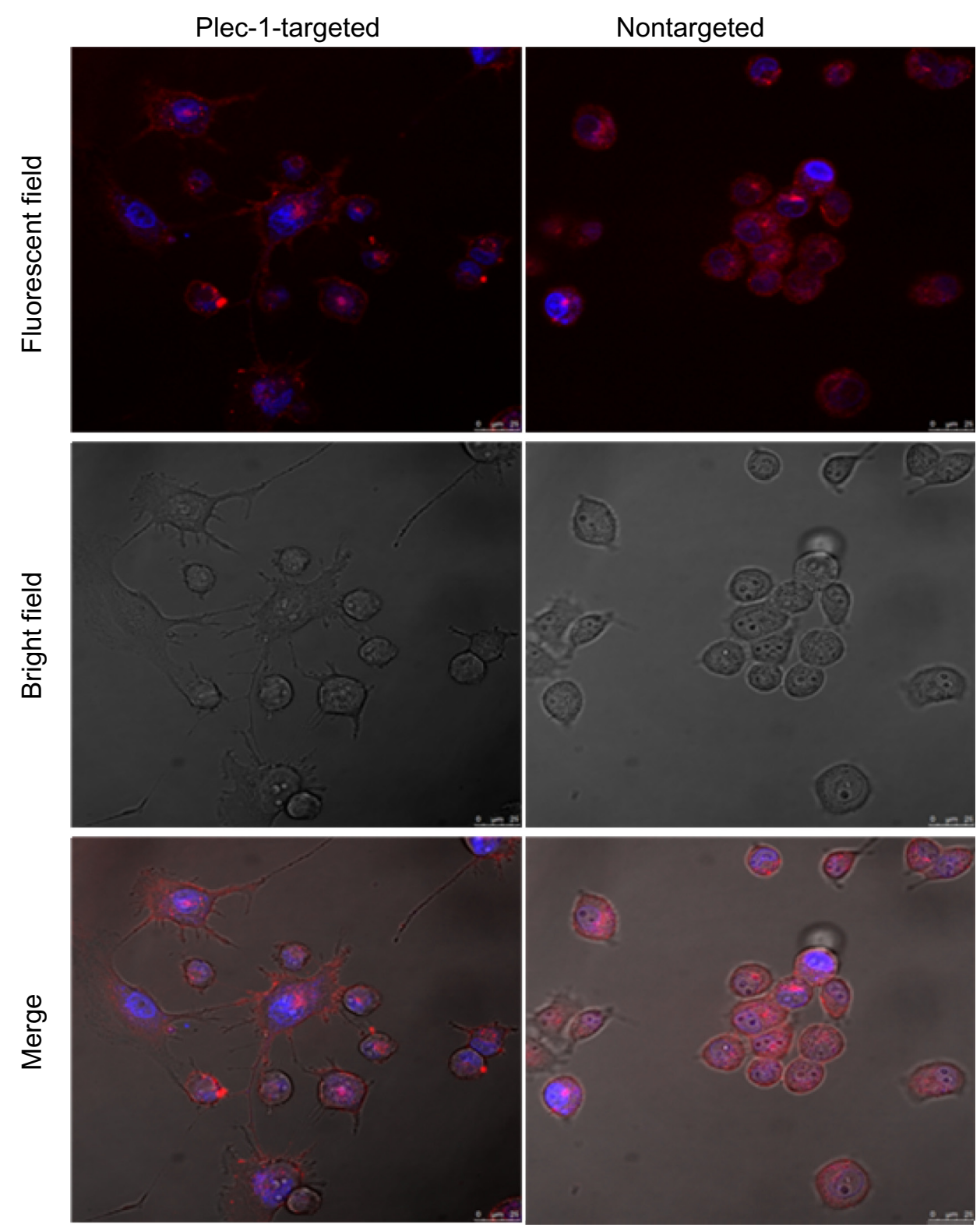

Figure S5 Panc-I cells (Plec-I-positive) were incubated with Plec-I-targeted and nontargeted dyebovine serum albumin surface functionalized hydrophobic superparamagnetic iron oxide nanoparticles for 30 minutes.

Notes: As the incubation time increases, cellular internalization of nanoparticles (in red) results in nonspecific signals for nontargeted dyebovine serum albumin surface functionalized hydrophobic superparamagnetic iron oxide nanoparticles conjugates. Nuclei were labeled by 4',6-diamidino-2-phenylindole in blue.

International Journal of Nanomedicine

\section{Publish your work in this journal}

The International Journal of Nanomedicine is an international, peerreviewed journal focusing on the application of nanotechnology in diagnostics, therapeutics, and drug delivery systems throughout the biomedical field. This journal is indexed on PubMed Central, MedLine, CAS, SciSearch ${ }^{\circledR}$, Current Contents ${ }^{\circledR} /$ Clinical Medicine,
Journal Citation Reports/Science Edition, EMBase, Scopus and the Elsevier Bibliographic databases. The manuscript management system is completely online and includes a very quick and fair peer-review system, which is all easy to use. Visit http://www.dovepress.com/ testimonials.php to read real quotes from published authors.

\footnotetext{
Submit your manuscript here: http://www.dovepress.com/international-journal-of-nanomedicine-journal
} 